\title{
Calcifying fibrous pseudotumor of the adrenal gland: A rare case report
}

\author{
TAO WU ${ }^{1 *}$, PINGYU ZHU $^{1 *}$, XI DUAN ${ }^{2}$, XUESONG YANG $^{1}$ and DONGLIANG LU ${ }^{1}$ \\ Departments of ${ }^{1}$ Urology and ${ }^{2}$ Dermatovenereology, Affiliated Hospital of North Sichuan Medical College, \\ Nanchong, Sichuan 637000, P.R. China
}

Received February 25, 2016; Accepted May 30, 2016

DOI: $10.3892 / \mathrm{mco} .2016 .944$

\begin{abstract}
Calcifying fibrous pseudotumor (CFP) is a rare benign soft tissue lesion. We herein present an extremely rare case of CFP of the adrenal gland. A 32-year-old male patient presented to our hospital with a tumor-like lesion in the area of the left adrenal gland detected by ultrasound during a routine check-up several days prior. Contrast-enhanced magnetic resonance imaging examination revealed a solitary, well-circumscribed mass, in close proximity to the medial arterial branch of the left adrenal gland. Histological examination revealed that the tumor consisted of sheets of varying amount of inflammatory cell infiltration; interstitial fibrosis and psammomatous calcifications were also observed. To the best of our knowledge, this is the third case of CFP occurring in the adrenal gland in the published literature. We herein report the findings of this case and review the two previously reported cases of CFP of the adrenal gland with valuable information.
\end{abstract}

\section{Introduction}

Calcifying fibrous pseudotumors (CFP) are rare, benign soft tissue neoplasms. These lesions are characterized by hypocellular, densely hyalinized collagenization with variably prominent mononuclear inflammatory infiltrate and scattered psammomatous and/or dystrophic calcification (1). The etiology of CFT is unknown. A few were considered to evolve from inflammatory myofibroblastic tumors (IMTs), but larger studies have failed to confirm this, and ultrastructural studies revealed fibroblastic features (2,3). CFPs do not have a propensity to metastasize, and are often treated by local excision with clear margins (4). Although there is a low risk for recurrence, consideration should be given to re-excision, should this occur.

Correspondence to: $\mathrm{Dr}$ Tao Wu, Department of Urology, Affiliated Hospital of North Sichuan Medical College, Nanchong, Sichuan 637000, P.R. China

E-mail: alhawking@163.com

*Contributed equally

Key words: calcifying fibrous pseudotumor, adrenal gland, case report
Prognoses have been observed to be excellent with surgical treatment alone (5).

The aim of the present study was to present a further case of CFP of the adrenal gland, and to provide useful information on this rare type of tumor.

\section{Case presentation}

A 32-year-old male presented to our hospital with a tumor-like lesion in the area of the left adrenal gland detected by ultrasound during a routine check-up several days prior. There were no clinical symptoms or positive signs on physical examination. There was no family history or relevant medical history. The adrenal hormones including catecholamines, cortisol, adrenocorticotropic hormone, renin, angiotensin and aldosterone, were all within the normal range. Contrast-enhanced magnetic resonance imaging revealed a solitary, well-circumscribed mass, sized $4.4 \times 4.6 \times 4.1 \mathrm{~cm}$, in close proximity to the medial arterial branch of the left adrenal gland. T1-weighted images showed a mixture of low and equal signal intensity. T2-weighted images with fat suppression showed areas of low and high signal intensity. On enhanced T1-weighted images, the mass exhibited significant heterogeneous enhancement (Fig. 1A). A kidney/ureter/bladder X-ray showed a large stone or calcified lesion in the left adrenal area (Fig. 1B). Other routine investigations, including a chest radiograph, were normal. The patient underwent left retroperitoneal laparoscopic adrenalectomy. Histological examination showed that the tumor consisted of sheets of varying amounts of inflammatory cells; interstitial fibrosis and psammomatous calcifications were also observed (Fig. 1C). Immunohistochemical examination revealed diffuse staining for CD34, CD31, cytokeratin, synapsin and partially for inhibin (Fig. 1D). The cells were negative for all other antibodies tested, including chromogranin A, calretenin and Ki67. These results confirmed the diagnosis of CFP of the adrenal gland. After treatment, the patient remained recurrence-free at 3 months.

\section{Discussion}

CFP is a rare benign lesion histologically characterized by dense hyalinization, fibrous proliferation, inflammatory infiltration by mononuclear cells and scattered psammomatous and/or dystrophic calcifications (6-8). A review of the literature 

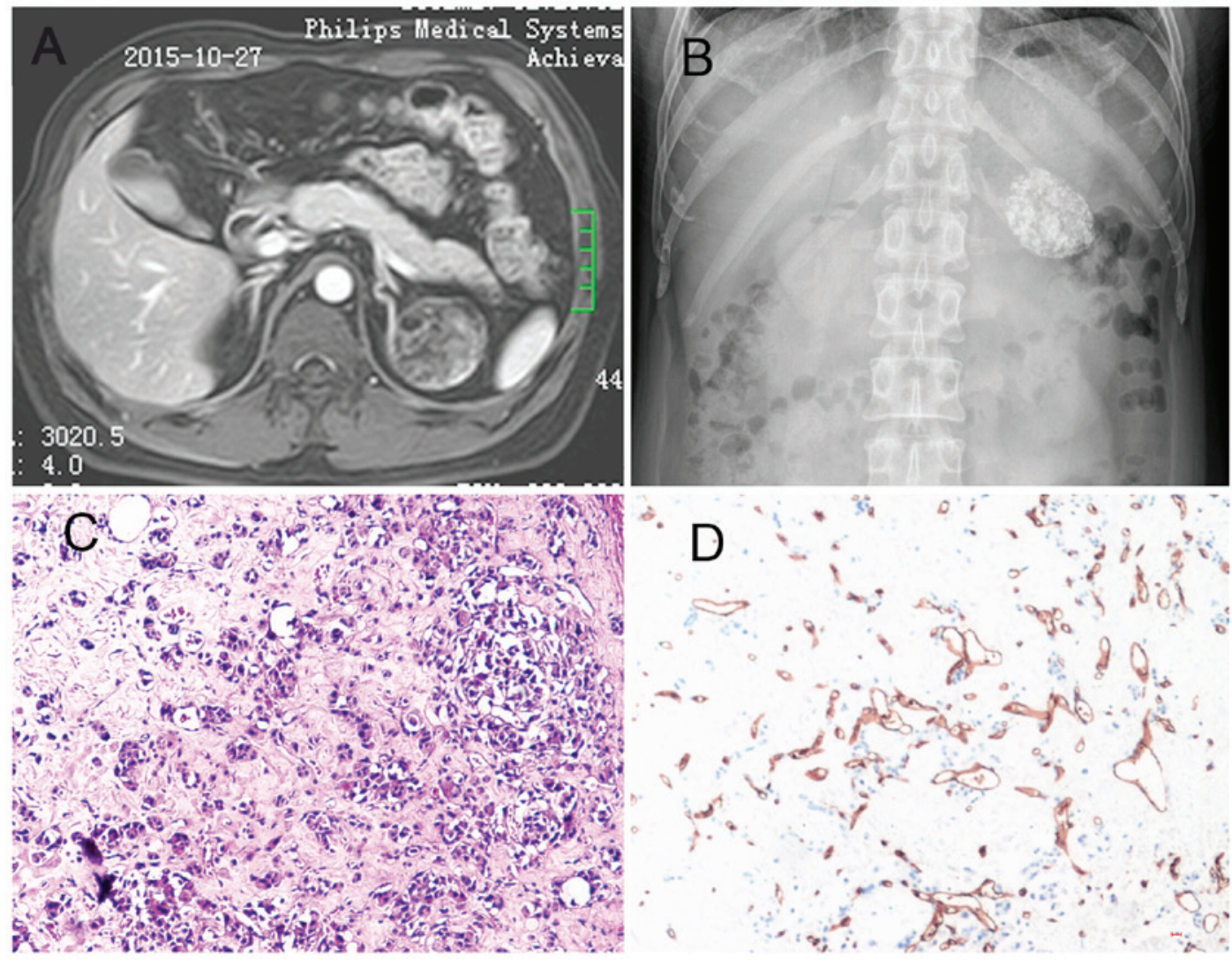

Figure 1. (A) Contrast-enhanced T1-weighted image showing a solitary, well-circumscribed mass with heterogeneous significant enhancement. (B) A kidney/ureter/bladder X-ray revealed a large stone or calcified lesion in the left adrenal area. (C) Histological section showing varying amounts of inflammatory cell infiltration, interstitial fibrosis and psammomatous calcifications (hematoxylin and eosin staining; magnification, x100). (D) Immunohistochemical staining showing CD34 reactivity (anti-CD34 immunoperoxidase staining; magnification, x100).

reveals that CFPs are rare, with the sites of involvement limited to the lung, mediastinum, myocardium, pleura and adrenal gland $(2,9-11)$. To the best of our knowledge, this is the third case of this type of lesion occurring in the adrenal gland published in the English literature. The previous two cases were reported in 2007 and $2001(12,13)$. One of those cases was a 10-year-old female patient with intermittent frontal headaches and a retroperitoneal mass. The pathological findings revealed a spindle-cell neoplasm forming irregular bundles and fascicles, without microscopic atypia. Chronic lymphoplasmocytic inflammatory cells forming prominent focal aggregates were also present (13); the other case was a 32-year-old female patient who presented with a chief complaint of vague abdominal discomfort. The identified lesion was well-circumscribed and was composed of dense, poorly cellular collagenous tissue, scattered spindle cells, an inflammatory infiltrate consisting of plasma cells and lymphocytes, and dystrophic calcifications (12). Following surgical resection, the second patient remained recurrence-free at 22 months; however, there are no follow-up data on the first case. CFP should be distinguished from other tumor occurring in the adrenal gland. The principal consideration in the differential diagnosis of CFP of the adrenal gland is IMT. Although originally described in the lung, IMT has also been recognized in a wide variety of extrapulmonary sites, including the adrenal gland $(7,14)$. The differentiation diagnosis between them has demonstrated chromosomal rearrangements involving the anaplastic lymphoma kinase gene located at 2p23 in a significant subset of cases (14). CFP should also be differentiated from other uncommon mesenchymal tumors that may originate in the adrenal gland, including benign peripheral nerve sheath tumors and solitary fibrous tumor (14). Immunohistochemistry is suitable for the purpose of differentiation, since CFP lacks S-100 protein reactivity (14). The aim of the present study was to present another case of CFP of the adrenal gland and provide useful information on this rare type of tumor.

\section{References}

1. Fetsch JF, Montgomery EA and Meis JM: Calcifying fibrous pseudotumor. Am J Surg Pathol 17: 502-508, 1993.

2. Nascimento AF, Ruiz R, Hornick JL and Fletcher CD: Calcifying fibrous 'pseudotumor': clinicopathologic study of 15 cases and analysis of its relationship to inflammatory myofibroblastic tumor. Int J Surg Pathol 10: 189-196, 2002.

3. Jaiswal SS, Agrawal A, Sahai K and Nair SK: Large retroperitoneal calcifying fibrous tumor. Med J Armed Forces India 69: 184,2013

4. Mangat A, Schiller C, Mengoni P, Reynolds C and Jeruss JS: Calcifying fibrous pseudotumor of the breast. Breast J 15: 299-301, 2009.

5. Maeda A1, Kawabata K and Kusuzaki K: Rapid recurrence of calcifying fibrous pseudotumor (a case report). Anticancer Res 22: 1795-1797, 2001.

6. Rosenthal NS and Abdul-Karim FW: Childhood fibrous tumor with psammoma bodies. Clinicopathologic features in two cases. Arch Pathol Lab Med 112: 798-800, 1988. 
7. Lau SK and Weiss LM: Calcifying fibrous tumor of the adrenal gland. Hum Pathol 38: 656-659, 2007.

8. Eftekhari F, Ater JL, Ayala AG and Czerniak BA: Case report: Calcifying fibrous pseudotumour of the adrenal gland. $\mathrm{Br} \mathrm{J}$ Radiol 74: 452-454, 2001.

9. Shinohara N, Nagano S, Yokouchi M, Arishima Y, Tabata K, Higashi M, Kitajima S, Yonezawa S and Komiya S: Bilobular calcifying fibrous pseudotumor in soleus muscle: A case report. J Med Case Rep 5: 487, 2011.

10. Minerowicz C, Jagpal S, Uppaluri L, Deen M and Langenfeld J: Calcifying fibrous pseudotumor of the pleura. Am J Respir Crit Care Med 192: e57-e58, 2015.

11. Özkan S, Demirağ F, Yekeler E and Karaoğlanoǧlu N: Calcifying fibrous pseudotumor of lungs. Turk J Med Sci 44: 901-903, 2014.
12. Chang JW, Kim JH and Maeng YH: Calcifying fibrous pseudotumor of the anterior mediastinum. Korean J Thorac Cardiovasc Surg 44: 318-320, 2011

13. Chan JK1, Cheuk W and Shimizu M: Anaplastic lymphoma kinase expression in inflammatory pseudotumors. Am J Surg Pathol 25: 761-768, 2001.

14. Van Dorpe J, Ectors N, Geboes K, D'Hoore A and Sciot R: Is calcifying fibrous pseudotumor a late sclerosing stage of inflammatory myofibroblastic tumor? Am J Surg Pathol 23: 329-335, 1999. 